\title{
Acknowledgement of Reviewers for Journal of Cross-Cultural Gerontology, 2021
}

Published online: 3 February 2022

(c) Springer Science+Business Media, LLC, part of Springer Nature 2022

Journal of Cross-Cultural Gerontology gratefully acknowledges the contributions of our 2021 reviewers. Thank you for your dedication both to the journal and to the field.

$\begin{array}{lll}\text { Abalos, Jeofrey } & \text { Fung, Sai-fu } & \text { Ong, Michelle } \\ \text { Ansello, Edward } & \text { Gangopadhyay, Jagriti } & \text { Park, Nan Sook } \\ \text { Babulal, Ganesh } & \text { Gnambs, Timo } & \text { Prasad, Ravi Durga } \\ \text { Baldassar, Loretta } & \text { Guest, M. Aaron } & \text { Rodriquez, Jason } \\ \text { Bear-Lehman, Jane } & \text { Huang, Chih-Mao } & \text { Salinas, Jennifer } \\ \text { Becot, Florence } & \text { Ikels, Charlotte } & \text { Saunders, Gabrielle } \\ \text { Berglund, Lisa } & \text { Intrieri, Robert } & \text { Saunders, Pamela } \\ \text { Black, Helen K. } & \text { Keimig, Rose } & \text { Sayegh, Philip } \\ \text { Boehnke, Klaus } & \text { Kleiber, Douglas } & \text { Seifert, Alexander } \\ \text { Bof de Andrade, } & \text { Lamb, Sarah } & \text { Shore, Linda } \\ \text { Fabiola } & \text { Li, Hong (Fujian Medical } & \text { Söderman, Mirkka } \\ \text { Burant, Christopher } & \text { University) } & \text { Tatarko, Alexander } \\ \text { Cantu, Phillip } & \text { Li, Hong (University of } & \text { Teerawichitchainan, } \\ \text { Chen, Yu-Chih } & \text { Illinois at Urbana-Champaign) } & \text { Bussarawan Puk } \\ \text { Cohen, Steven } & \text { Li, Yawen } & \text { Tezcan, Tolga } \\ \text { Cox, Carole } & \text { Ludlow, Kristiana } & \text { Thang, Leng Leng } \\ \text { De La Cruz, Pablo } & \text { Mair, Christine } & \text { Timonen, Virpi } \\ \text { Dekker, Karien } & \text { Miyawaki, Christina } & \text { Tiselius, Elisabet } \\ \text { Dintrans, Pablo } & \text { Moeke-Maxwell, Tess } & \text { Van der Geest, Sjaak } \\ \text { Villalobos } & \text { Molony, Sheila } & \text { Victor, Christina } \\ \text { Dsouza, Sebestina } & \text { Moreno, Ximena } & \text { Waters, William } \\ \text { DuongTran, Paul } & \text { Motel-Klingebiel, Andreas } & \text { Wen, Ming } \\ \text { Feng, Qiushi } & \text { Müller, Nicole } & \text { Zhang, Weiguo } \\ \text { Fu, Yuanyuan } & \text { Nielsen, T. Rune } & \\ & & \end{array}$

Publisher's Note Springer Nature remains neutral with regard to jurisdictional claims in published maps and institutional affiliations. 